\title{
EXPERIMENTAL STUDIES OF UNPROTECTED AND PROTECTED STEEL STRUCTURES UNDER FIRE
}

\author{
Véronique Saulnier ${ }^{\mathrm{a}}$, Sébastien Durif ${ }^{\mathrm{a}}$, Abdelhamid Bouchaïr ${ }^{\mathrm{a}}$, Philippe Audebert ${ }^{\mathrm{a}}$, Mohamed \\ Lahmar $^{\mathrm{b}}$ \\ ${ }^{a}$ Blaise Pascal University, Institut Pascal axe Mechanical,Material,Struture, Clermont-Ferrand, France \\ ${ }^{\mathrm{b}}$ Blaise Pascal University, Polytech, Clermont-Ferrand, France
}

\begin{abstract}
Preliminary fire experimental tests have been carried out on steel plates protected with intumescent coating.

The tests evaluated and measured the heating of steel plates with various geometrical configurations: shape, size. A first test on an equal leg angle section showed interesting results with different coating expansion on the faces of the section.

The second test campaign is done with different protections to compare behaviors of four steel plates. The first objective is to set up an experimental protocol for future tests. Those results are used to evaluate analytical prevision of steel plate temperature with and without fire protection.
\end{abstract}

KEYWORDS: intumescent painting, fire protection, steel structures, fire behavior.

\section{INTRODUCTION}

Intumescent coating delays the heating process of steel. It is used for the fire protection of steel and concrete structures. The main advantage of this type of protection is that it doesn't alter the architectural aspect and the mechanical properties of the structure. However, the current European standards give no prescription rules to predict the efficiency of the protection. Indeed, manufacturers give some prescriptions for the coating thickness but without any material properties. Furthermore, this material is complex as it expands during the elevation of gas temperature. Thus, several researches have been carried out to study the behavior of this protection under elevated temperature and the influence of different parameters.

Tests were performed on cellular beams by Collin Bailey, ten years ago (Bailey 2004). It was also the subject of Gillet's thesis (Gillet 2009). All papers conclude that it is necessary to make new investigations. Other tests were carried out recently on different columns (Kolsek and Cesarek 2015). Many articles give models of intumescent painting (Buckmaster and Anderson 1986), but it is necessary to be able to calculate the steel heating.

Other fire protections can be used, like gypsum plasterboards. Even the behavior of plasterboard under fire exposure reveals complex mechanisms (Wakili et al. 2007) and usually, it is difficult to predict with accuracy its behavior under elevated temperature and thus, the temperature of the protected structure.

The aim of this work is to improve the knowledge on the behavior and characteristics of different fire protections under high temperatures and measure steel heating. Therefore, experimental tests were carried out on different steel plates in a furnace. Two tests campaign were performed, the first one focused on intumescent coating and the influence of different geometrical parameters on its behavior.

The second test studied steel plates unprotected, protected with plasterboard and also with intumescent coating. The aim was to compare elevation of temperature of the different steel plates in function of the fire protection.

The experimental measurements are compared to analytical formula from European standard, EN1993-1-2 (Eurocode, 2005).

The purpose of these tests is to observe and to compare theoretical and experimental values with the aim of testing new solution of fire protection for steel structures 


\section{EXPERIMENTAL SET-UP}

The experimental tests have been led in a furnace made of cellular concrete. Two oil burners have been used. The tests led on steel plates are presented in Figure 1 and described hereafter.

\subsection{First test campaign}

This test campaign implied two successive fire tests led on different steel plates. The first test studied the development of intumescent coating on simple plates to evaluate its efficiency by comparing the steel temperatures with unprotected plates. In the first test, there were: three plates with 5, 10 and $20 \mathrm{~cm}$ square. Furthermore, a protected L section had been tested to study the influence of corners, exposition and different kinds of singularities. A second test focused on four steel plates with specific shapes, like circular holes and hexagonal openings.

Samples were painted with $0.4 \mathrm{~mm}$ thick water-based intumescent painting.

\subsection{Second test campaign}

Three materials are involved in this experimental test campaign; there are two steel plates covered by intumescent coating, plasterboard and a steel plate unprotected. All plates are 10 centimeter square. Two plates are protected with intumescent coating with respectively 0.375 and $0.525 \mathrm{~mm}$ of painting thickness. The last plate is covered with plasterboard $12.5 \mathrm{~mm}$ thick.

The plate unprotected is used as a reference. Furthermore, contrary to the painted, the plasterboard thickness remains constant during the fire exposure. The aim was evaluating the temperature evolution of a steel plate protected with a constant thickness of material that has well-known properties. The furnace temperature has been measured with four thermoplates located in the furnace (Fig. 1 (b)). The observation of both measurements confirms that the temperature in the furnace remains homogenous. It increases for more than one hour. Thermocouples $\mathrm{K}$ which measure the temperature of plates are welded on and under samples.

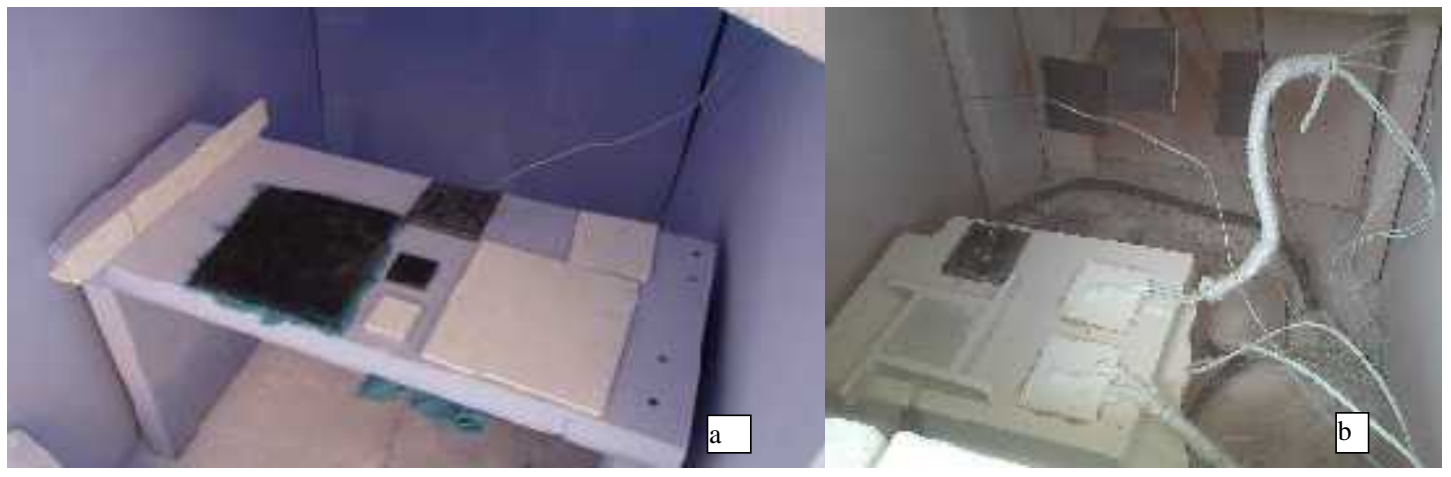

Fig. 1. Disposal of samples in the furnace phase 1(a) and 2(b)

\section{MATERIAL PROPERTIES}

\subsection{Steel and plasterboard behavior}

Theoretical elevation of temperature of steel unprotected and steel with plasterboard is given through equations from Eurocode 3 (Eurocode, 2005). However, gypsum plasterboard has complex behaviour under fire exposure. Even if it conserves a constant thickness, the thermal properties vary during the elevation of temperature with especially endothermic reaction due to water phase change in the plasterboard (Wakili et al., 2007).

\subsection{Intumescent painting mechanism}

Intumescent painting is a coating which expands with the increase of temperature. The development of the foam structure is a complex chemical process. The painting begins to absorb the flow of heat. An endothermic pyrolysis reaction takes place and water vaporizes. Gas bubbles rising to the surface will cause swelling (Duquesne et al., 2012). This phase is called viscous layer. If the 
temperature continues to rise it will form the char layer that corresponds to the charring of paint. (Zhan et al., 2012) (Yew et al., 2012)

\section{TEST FIRST PHASE RESULTS AND OBSERVATIONS}

\subsection{Steel plates}

First tests give temperature rise curves of the different samples (Fig.3). The development of a foam structure and char layer expansion has been observed. This development varies from one surface to the other. The foam of the smallest plate is more developed (Fig.2) and the foam of the medium plate is more developed near the furnace wall. (Fig.2). To conclude about this first test, the size of the plate seems to have an influence on the development of the foam and char layer structure. But the position in the furnace seems also to have an influence on the development of the protection.

In all cases the foam structure has a thickness of about two centimeters. The development has a ratio of 25 according to the manufacturer. It seems that the proximity of an edge has an influence on the foam development but it is difficult to quantify with this test.

The curves given in Figure 3 reveal the beginning of the insulation process of the intumescent coating. It can be seen also that the theoretical values for the unprotected plates are upper about $100^{\circ} \mathrm{C}$ with regard to the experimental results.

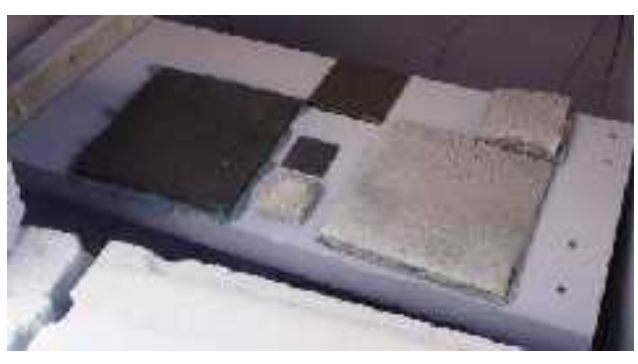

Fig. 2. Plates after firth test

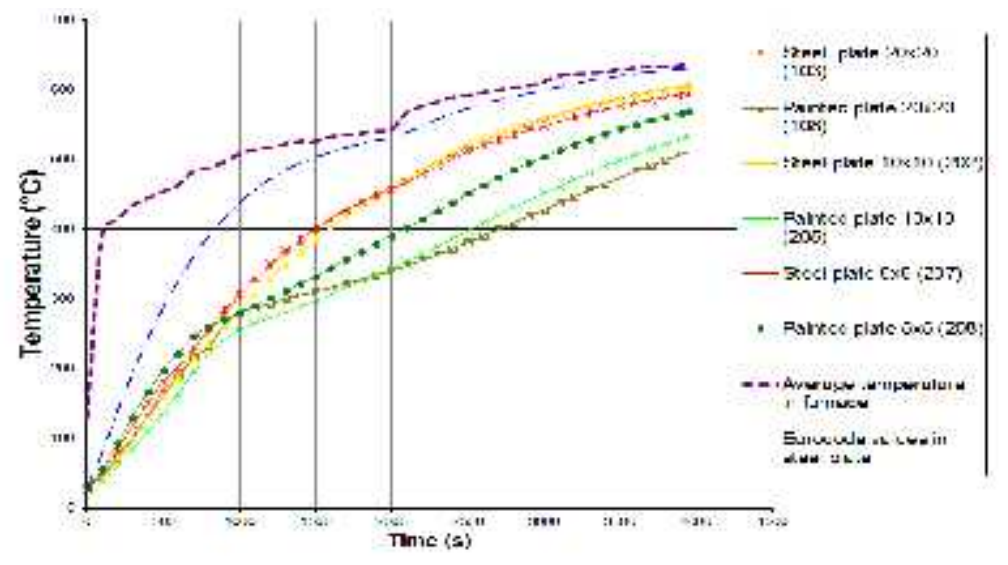

Fig. 3. Experimental and theoretical curves

\section{2 equal leg angle observations}

It can be observed on the equal leg angle sample that the foam is thinner on the vertical surfaces in comparison to the horizontal face (Fig 4).Furthermore, the development of the foam did not fill scratches formed on the L-section (vertical face). Besides painting on edges did not almost develop.

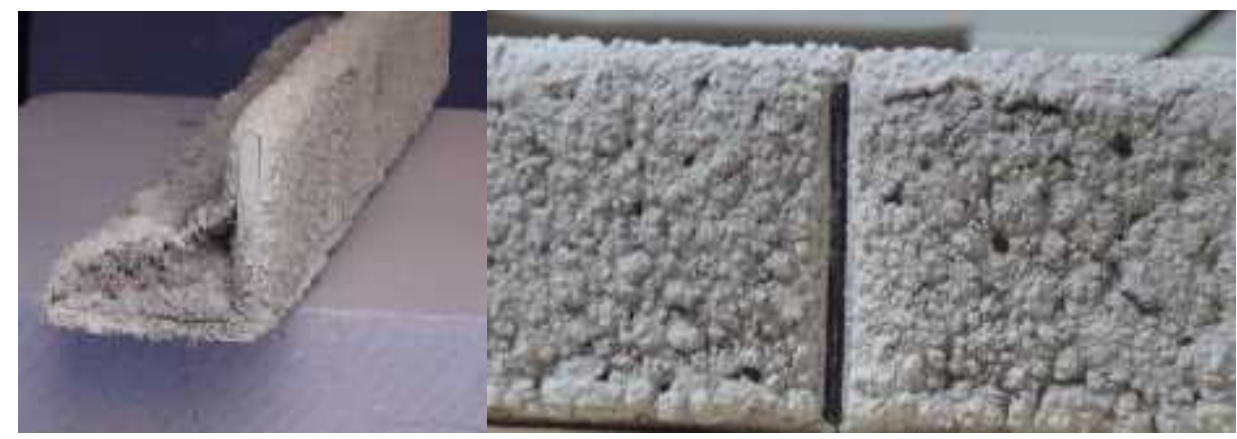

Fig. 4. L-section after firth test 


\section{TESTS PHASE TWO RESULTS AND OBSERVATIONS}

\subsection{Measures and comparisons}

Expansion mechanism of intumescent coating is the same as the previous test. It can be observed in Figure 7 that the curves of the temperature measured on the plates with intumescent coating have an inflection. Thus, steel temperature increase slower compared to the unprotected steel plate, especially beyond $250^{\circ} \mathrm{C}$.

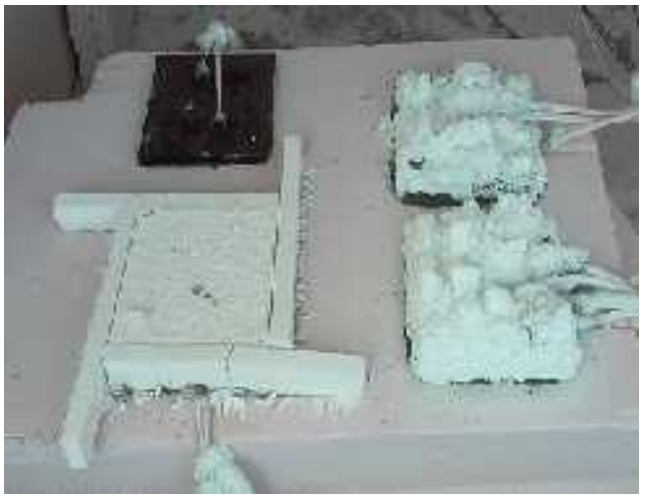

Fig. 5. Samples in the furnace after test

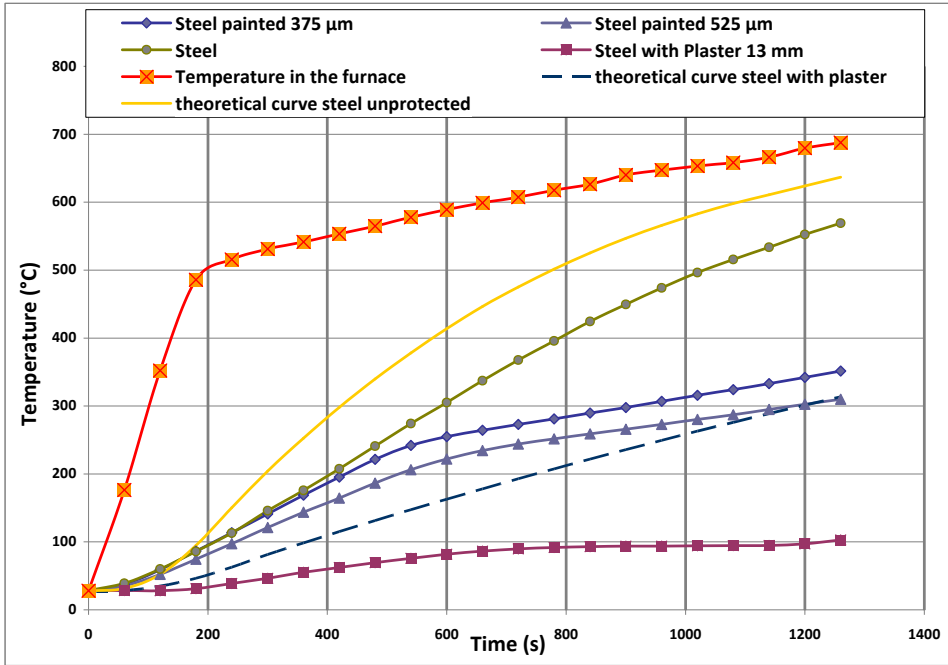

Fig. 6. Curves evolution of temperature

At the beginning, painting plates react like unpainted steel. But temperature of plate with $525 \mu \mathrm{m}$ of painting increases already slower. A consequent thickness of paint protects steel from the beginning. But the protection is less important than after foam development. When steel temperature overtakes $250^{\circ} \mathrm{C}$, steel painting temperature increases slower than unprotected steel. Painting thickness also has an influence on the steel heating.

For estimated heating protection, experimental curves are compared with Eurocode curves for a constant thickness. (Fig 7)

Theoretical curves were obtained by taking a thermal conductivity of $0.23 \mathrm{~W} / \mathrm{m} . \mathrm{K}$ and with a constant thick estimated with a ratio of 25 according to the indications of the manufacturer. Experimental curves are near of theoretical curves but not exactly the same (Fig.7). Nevertheless, this method allows estimating the thermal protection during viscous phase. (Tests stay under char phase). Thermal Conductivity value is high enough so that the experimental curve stays always under theoretical curve in all cases. This method is less precise than that used in the studies of Kolsek and Cesarek (2015) but it gives simply and fast results.
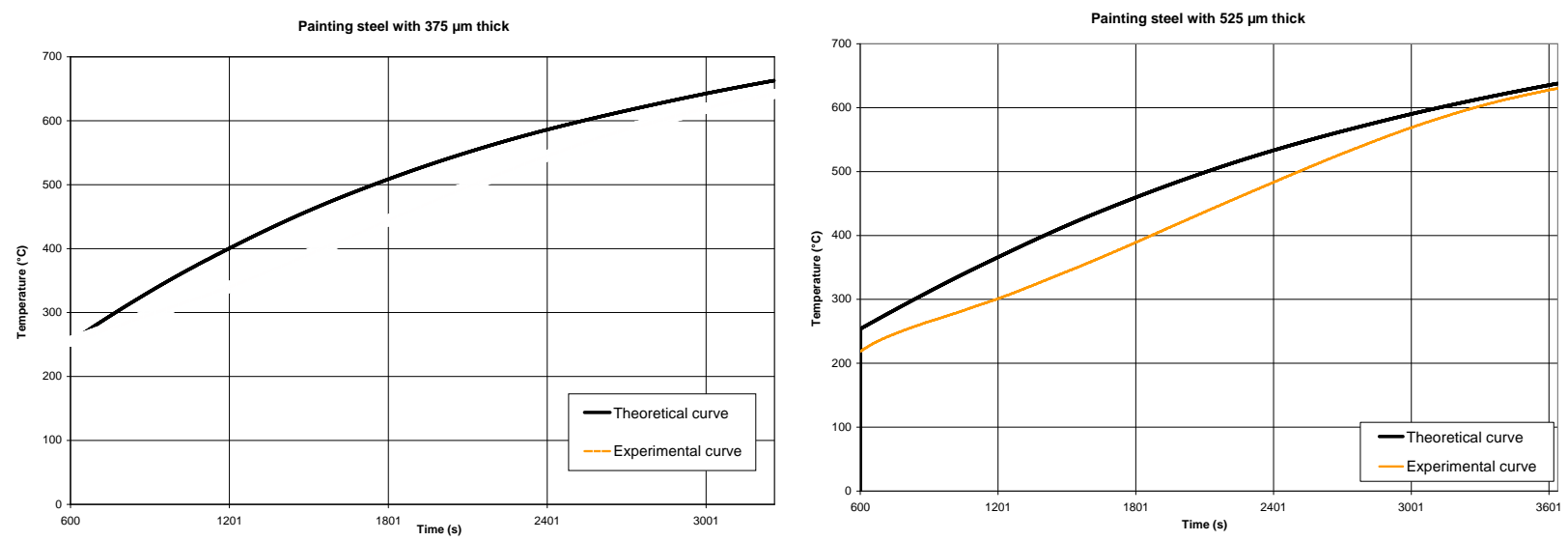

Fig. 7. Curves evolution of temperature for steel painting after $250^{\circ} \mathrm{C}$ 


\subsection{Case of plasterboard}

Plasterboard was chooses because it has a constant thickness and it is fireproof. It is also a material usually used in the construction and cheap. The main purpose is to have a material the properties of which are perfectly known to be able to test the formulae of the Eurocode

Plasterboard behavior is like descriptions in literature. . The temperature curve is well below the theoretical curve. But before $100^{\circ} \mathrm{C}$, for a similar curve must take a thermal conductivity of 0.08 $\mathrm{W} / \mathrm{m} . \mathrm{K}$ instead of $0.25 \mathrm{~W} / \mathrm{m} . \mathrm{K}$. The experimental curve is quickly inflected around $100^{\circ} \mathrm{C}$. This is due to the water phase change of the material, (Wakili et al., 2007).

After twenty minutes, the curve grows up faster (Fig 8 (a)).
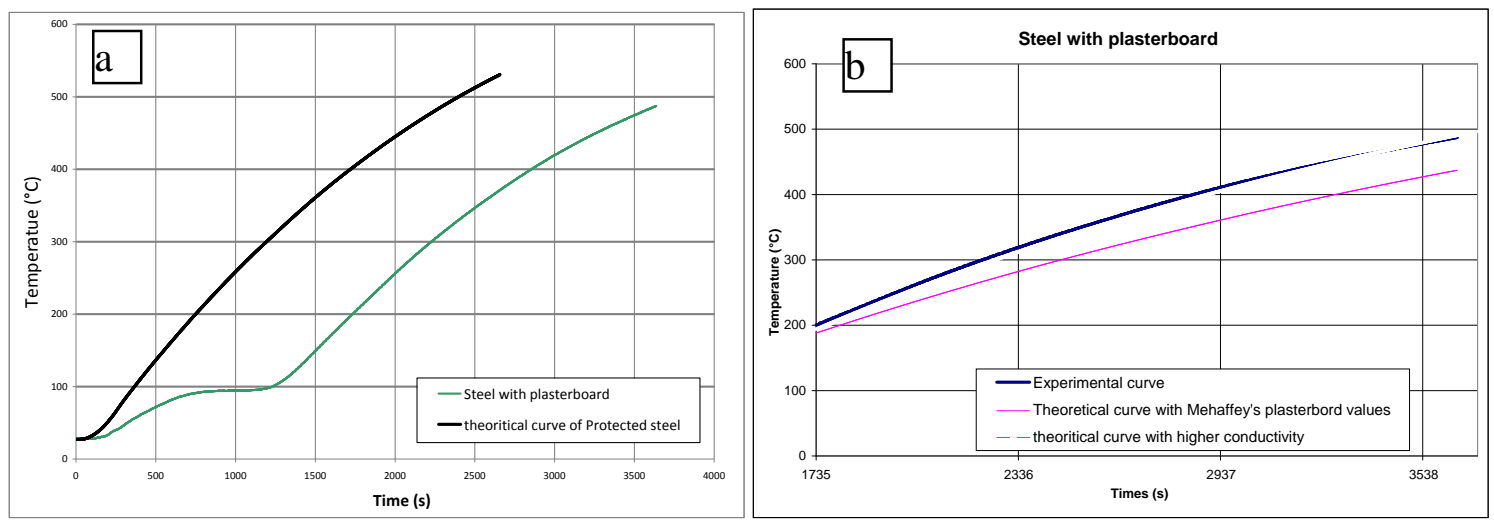

Fig. 8. Curves for plasterboard

After water vaporization, experimental curve has the same form as the theoretical curve, thermal properties of gypsum according Mehaffey et al (1994) and Thomas (2002).

If the simulation is done with a steel departure temperature of $200^{\circ} \mathrm{C}$, the theoretical curve appears similar to the experiment curve (Fig 8 (b)). And with a thermal conductivity of $0.16 \mathrm{~W} / \mathrm{m} . \mathrm{K}$, experimental and theoretical curves are confused. At the end, with the steel expansion, plasterboard protection cracked and protection efficiency may be affected.(Axenenko \& Thorpe 1996). However test results don't show significant curve inflection.

Plasterboard turns out to be an effective protection against steel heating.

\section{CONCLUSIONS AND OUTLOOKS}

The tests allowed the observation of the foam development. After $250^{\circ} \mathrm{C}$ protected plates heat up less than unprotected ones. For unprotected plates results are near of theoretical results. Test made on equal leg angle allowed observing a difference in the development of the foam between horizontal and vertical surfaces. Further tests on IPE profiles may confirm this behavior.

Tests with plasterboard showed a complex behavior. But the delay of heating due to the evaporation of the water is very interesting to delay steel heating. .So fire resistance plasterboard opens interesting perspectives. Further experiments are to be prepared in order to measure the evolution of plasterboard conductivity during temperature elevation in order to have better data for theoretical and numerical calculations. It exists fireproof plasterboard but it is not necessary that plasterboard has a mechanical resistance. However this solution is not excluded from future tests. It is also necessary to make others tests on intumescent coating to confirm conductivity values.

\section{ACKNOWLEDGMENTS}

Thanks to all those who permit the realization of these tests. A gratefully acknowledgment to Myriam Moissaing and Daniel Robin for their help in conducting experiments.

\section{REFERENCES}

AFNOR, N. NF EN 1363-1. Fire resistance test-Part, 1. 
Ang, C. N., \& Wang, Y. C. (2009). Effect of moisture transfer on specific heat of gypsum plasterboard at high temperatures. Construction and Building Materials, 23(2), 675-686.

Axenenko, O., \& Thorpe, G. (1996). The modelling of dehydration and stress analysis of gypsum plasterboards exposed to fire. computational materials science, 6(3), 281-294.

Bailey, C. (2004). Indicative fire tests to investigate the behaviour of cellular beams protected with intumescent coatings. Fire Safety Journal, 39(8), 689-709.

Buckmaster, J., Anderson, C., \& Nachman, A. (1986). A model for intumescent paints. International journal of engineering science, 24(3), 263-276.

Duquesne, S., Magnet, S., Jama, C., \& Delobel, R. (2005). Thermoplastic resins for thin film intumescent coatings-towards a better understanding of their effect on intumescence efficiency. Polymer Degradation and Stability, 88(1), 63-69.

Eurocode, E. C. (Ed.). (2005). EN 1993-1-2: Eurocode 3: Design of Steel Structures. General rulesStructural fire design. Brussels: European Committee for Standardization. DD ENV, 1-2.

Franssen, J. M., \& Zaharia, R. (2005). Design of Steel Structures subjected to Fire. Background and Design Guide to Eurocode 3. Les Editions de l'Universite de Liege.

Gerlich, H. (2004). Calcination of Gypsum Plasterboard under Fire Exposure (Doctoral dissertation, University of Canterbury).

Gillet, M. (2009). Analyse de systèmes intumescents sous haut flux: modélisation et identification paramétrique (Doctoral dissertation, Université d'Angers).

Kolšek, J., \& Češarek, P. (2015). Performance-based fire modelling of intumescent painted steel structures and comparison to EC3. Journal of Constructional Steel Research, 104, 91-103.

Mehaffey, J. R., Cuerrier, P., \& Carisse, G. (1994). A model for predicting heat transfer through gypsum board/wood stud walls exposed to fire. Fire and Materials, 18(5), 297-305.

Rahmanian, I. (2011). Thermal and Mechanical Properties of Gypsum Boards and Their Influences on Fire Resistance of Gypsum Board Based Systems.

Rahmanian, I., \& Wang, Y. (2009). Thermal Conductivity of Gypsum at High Temperatures-A Combined Experimental and Numerical Approach. Acta Polytechnica, 49(1).

Thomas, G. (2002). Thermal properties of gypsum plasterboard at high temperatures. Fire and Materials, 26(1), 37-45.

Wakili, K. G., Hugi, E., Wullschleger, L., \& Frank, T. H. (2007). Gypsum board in fire-modeling and experimental validation. Journal of Fire Sciences, 25(3), 267-282.

Yew, M. C., \& Sulong, N. R. (2012). Fire-resistive performance of intumescent flame-retardant coatings for steel. Materials \& Design, 34, 719-724.

Zhang, Y., Wang, Y. C., Bailey, C., \& Taylor, A. P. (2012). Global modelling of fire protection performance of intumescent coating under different cone calorimeter heating conditions. Fire Safety Journal, 50, 5162. 